\title{
A hypothetical approach on gender differences in cancer diagnosis
}

\author{
Katrin Sak \\ NGO Praeventio, Tartu, Estonia
}

In parallel to the continual increase in cancer diagnosis all over the world, the number of studies on tumorigenesis is constantly rising. However, there are still no curative treatment options available for patients, particularly for those suffering from advanced metastatic malignancies, and many aspects related to carcinogenesis process have remained mysterious even in the $21^{\text {st }}$ century. Based on the global cancer statistics, one in two men and one in three women will be diagnosed with some type of invasive cancer during their lifetime $e^{[1-3]}$, showing that men are more susceptible to cancer than women. On the other hand, it is also well accepted that people develop a certain number of mutations that could result in cancer each day, but by virtue of strong immune system, the body is able to eliminate this threat. ${ }^{[4]}$ Therefore, the question remains, why one person is diagnosed with cancer while another is spared, or more generally, why men are more prone to get a cancerous disease as compared to women. Despite intensive efforts done on the understanding of the mechanisms of carcinogenesis and spread of malignant tumors, we are still unable to explain this intriguing gender-related phenomenon today. It is even proposed to focus on the healthy individuals rather than patients carrying malignant neoplasms to clarify specific molecular features, which could prevent the development of malignancies and provide protection against invasive cancers. ${ }^{\left[{ }^{[j]}\right.}$ Indeed, two of three humans remain unaffected from this devastating disease.

Numerous autopsy studies have reported a high prevalence of incidental cancer cases in people who died of other causes, ${ }^{[0-8]}$ altogether showing that certain proportion of malignant neoplasms remains forever latent and undiagnosed. This phenomenon is particularly known in the case of prostatic tumors. It is even proposed the concept "to live in harmony with the tumor". ${ }^{[9]}$

Based on all this knowledge, it can be hypothesized that gender differences observed in cancer diagnosis all over the world do not indicate the real variation in the incidence of malignant neoplasms in women and men, but rather reflects the proportion of those cancers that are invasive and progressive. In other words, half of males and one third of females get invasive, aggressive tumors in their lifetime, while the incidence of all neoplasms, including latent malignant lesions, which are never clinically diagnosed, is probably similar for both genders. It means that the processes of progression and metastasis are dependent on gender and sex-specific factors, but not the malignant transformation and initiation of cancer itself.

The ability to metastasize is one characteristic feature of malignant tumors. Cancer invasion and metastasis are highly regulated by matrix metalloproteinases (MMPs), mainly MMP-2 and MMP-9, which are type IV collagenases. MMPs are capable to degrade extracellular matrix proteins, thereby promoting invasion and migration of tumoral cells. Compounds suppressing the activity or expression of these enzymes are important in antimetastatic therapy and restricting cancer spread. The same enzymes are also involved in other pathological conditions in the human body, including certain connective tissue disorders, which reveal clear gender differences in 
their incidence. For example, systemic sclerodermas, characterized by excessive tissue matrix accumulation due to the reduced levels of MMPs, have significant female preponderance. ${ }^{[10,11]}$ The question further arises, whether there is any correlation between clinical incidence rate of fibroproliferative disorders with abnormal activity of MMPs and cancer; or in other words, what is the tendency of cancer incidence among people suffering from connective tissue disorders regarding the general population. At the moment, it could just be presumed that individuals with lower levels of MMPs, being more prone to connective disorders related to matrix accumulation, are less susceptible towards invasive metastatic cancers. Therefore, the strong female prevalence reported in many connective tissue diseases might provide an explanation to the observed lower rate of invasive metastatic cancer cases among women as compared to men. This hypothetic presumption does not exclude the similar incidence rate of small latent undiagnosed malignant neoplasms in both genders.

Furthermore, as connective tissue disorders are often heritable and occur in family members, they could, at least partially, be associated with familial cancer syndrome without an established genetic background. It is well known that malignant neoplasms occur in some families more often than it would be expected by chance and reasons for this phenomenon have mostly remained unknown.

In addition, it is possible that findings about suppressive activities of vitamin $\mathrm{C}$ in carcinogenesis might mainly reflect the restrictive effects of this natural compound on the activity and expression of MMPs, thereby suppressing invasion, migration and metastasis, as it is well known that vitamin $\mathrm{C}$ is a strong inhibitor for both MMP-2 and MMP-9. ${ }^{[12,13]}$

Several molecular studies support the speculative statement presented in this commentary article. In fact, a number of results have shown that female steroid hormones estradiol and progesterone can downregulate the expression of MMPs and reduce the activity of these enzymes in different malignant as well as non-malignant tissues, including human choriocarcinoma cells, endometrial cancer cells and hormone-positive breast cancer cells, ${ }^{[14-19]}$ thus providing protection against metastasis of initial tumors and impeding dissemination of cancerous cells. Moreover, it was shown that breast cancer patients with no expression of estrogen or progesterone receptors but positive for MMP-2 had worst disease-free and overall survival rates. ${ }^{[20]}$ On the other hand, androgen as male steroid hormone can stimulate MMP-2 expression in human prostate cancer cells; ${ }^{[21]}$ and elevated levels of androgen receptors, MMP2 and MMP-9 in human hepatocellular carcinoma tissues are associated with higher invasive potential of these liver tumors. ${ }^{[22]}$

Therefore, based on the hypothesis proposed in the current article, small latent tumors may not be so dangerous as it is generally assumed. People can live with their undiagnosed tumors for years and these latent localized neoplasms can be detected only within the autopsy after death for any other reason. Human organism can apparently adapt to live with such latent malignant lesions and intervention in these neoplasms might be accompanied by overtreatment and poisoning of the body with adjuvant cytotoxic chemotherapy or radiotherapy. It would be just exciting to speculate about the huge amount of such small lesions detected incidentally in "healthy" people undergoing positron emission tomography and computed tomography (PET-CT) scanning. It is probable that these small lesions, which have persisted unchanged for years unknowingly in their hosts, occur equally in both men and women, but men are just more susceptible to invasion and migration of these neoplasms, while women are somewhat more protected against metastatic spread. Moreover, pathological conditions related to excessive extracellular matrix might provide benefit in the prevention of cancer spread. In any case, it is clear that we should focus much more on adjuvant antimetastatic treatment options in the future, but not only administering cytotoxic drugs.

Altogether, it is possible that not the incidence of all malignant lesions, but rather the rate of invasive and metastatic cancerous neoplasms is different among females and males. Further studies should prove or disprove this hypothetical presumption with the ultimate aim to better evaluate the prognostic criteria and provide the most appropriate approach for the management of malignant neoplasms.

\section{Conflict of Interest}

None declared.

\section{REFERENCES}

1. Birch JC, Khatri G, Watumull LM, Arriaga YE, Leyendecker JR. Unintended consequences of systemic and ablative oncologic therapy in the abdomen and pelvis. Radiographics 2018; 38: 1158-79.

2. Sharmer DL. Medical student exposure to cancer patients whilst on clinical placement: A retrospective analyses of clinical log books. J Cancer Educ 2019; 34: 671-6.

3. Herrmann A, Hall A, Proietto A. Using the health belief model to explore why women decide for or against the removal of their ovaries to reduce their risk of developing cancer. BMC Womens Health 2018; 18: 184.

4. Pardoll D. Cancer and the immune system: Basic concepts and targets for intervention. Semin Oncol 2015; 42: 523-38.

5. Klein G. Toward a genetics of cancer resistance. Proc. Natl Acad Sci U S A 2009; 106: 859-63. 
6. Bell KJ, Del Mar C, Wright G, Dickinson J, Glasziou P. Prevalence of incidental prostate cancer: A systematic review of autopsy studies. Int J Cancer 2015; 137: 1749-57.

7. Jahn JL, Giovannucci EL, Stampfer MJ. The high prevalence of undiagnosed prostate cancer at autopsy: implications for epidemiology and treatment of prostate cancer in the Prostate-specific Antigen-era. Int J Cancer 2015; 137: 2795-802.

8. Thomas ET, Del Mar C, Glasziou P, Wright G, Barratt A, Bell KJL. Prevalence of incidental breast cancer and precursor lesions in autopsy studies: a systematic review and meta-analysis. BMC Cancer 2017; 17: 808.

9. Abaza MS, Orabi KY, Al-Quattan E, Al-Attiyah RJ. Growth inhibitory and chemo-sensitization effects of naringenin, a natural flavanone purified from Thymus vulgaris, on human breast and colorectal cancer. Cancer Cell Int 2015; 15: 46.

10. Young-Min SA, Beeton C, Laughton R, Plumpton T, Bartram S, Murphy G, et al. Serum TIMP-1, TIMP-2, and MMP-1 in patients with systemic sclerosis, primary Raynaud 's phenomenon, and in normal controls. Ann Rheum Dis 2001; 60: 846-51.

11. D`Amico F, Skarmoutsou E, Mazzarino MC. The sex bias in systemic sclerosis: on the possible mechanisms underlying the female disease preponderance. Clin Rev Allergy Immunol. 2014; 47: 334-43.

12. Pfeffer F, Casanueva E, Kamar J, Guerra A, Perichart O, Vadillo-Ortega F. Modulation of 72-kilodalton type IV collagenase (Matrix metalloproteinase-2) by ascorbic acid in cultured human amnion-derived cells. Biol Reprod 1998; 59: 326-9.

13. Hernandez Guerrero CA, Vazquez Vela ME, Herrerias Canedo T, Flores Herrera H, Meraz Cruz N. Vitamin C decreases MMP-9 synthesis induced by hydrogen peroxide in an in vitro chorioamniotic membrane model. Ginecol Obstet Mex 2006; 74: 3-12.

14. Goldman S, Lovett DH, Shalev E. Mechanisms of matrix metalloproteinase-2 (mmp-2) transcriptional repression by progesterone in jar choriocarcinoma cells. Reprod. Biol Endocrinol 2009; 7: 41.
15. Waheed S, Dorjbal B, Hamilton CA, Maxwell GL, Rodriguez GC, Syed V. Progesterone and calcitriol reduce invasive potential of endometrial cancer cells by targeting ARF6, NEDD9 and MT1-MMP. Oncotarget 2017; 8: 113583-97.

16. Nilsson UW, Garvin S, Dabrosin C. MMP-2 and MMP-9 activity is regulated by estradiol and tamoxifen in cultured human breast cancer cells. Breast Cancer Res Treat. 2007; 102: 253-61.

17. Mahmoodzadeh S, Dworatzek E, Fritschka S, Pham TH, Regitz-Zagrosek V. 17beta-Estradiol inhibits matrix metalloproteinase-2 transcription via MAP kinase in fibroblasts. Cardiovasc Res 2010; 85: 719-28.

18. Yin H, Wan Q, Tian Y, Zhao B, Deng Y. Female hormone 17 $\beta$-estradiol downregulated MMP-2 expression and upregulated A1P1 expression in human corneal stromal cells. Cell Biochem Biophys 2018; 76: 265-71.

19. Zong W, Meyn LA, Moalli PA. The amount and activity of active matrix metalloproteinase 13 is suppressed by estradiol and progesterone in human pelvic floor fibroblasts. Biol Reprod 2009; 80: 367-74.

20. Ramos EA, Silva CT, Manica GC, Pereira IT, Klassen LM, Ribeiro EM, et al. Worse prognosis in breast cancer patients can be predicted by immunohistochemical analysis of positive MMP-2 and negative estrogen and progesterone receptors. Rev. Assoc Med Bras (1992) 2016; 62: 774-81.

21. Liao X, Thrasher JB, Pelling J, Holzbeierlein J, Sang QX, Li B. Androgen stimulates matrix metalloproteinase-2 expression in human prostate cancer. Endocrinology 2003; 144: 1656-63.

22. Zhang Y, Shen Y, Cao B, Yan A, Ji H. Elevated expression levels of androgen receptors and matrix metalloproteinase- 2 and -9 in 30 cases of hepatocellular carcinoma compared with adjacent tissues as predictors of cancer invasion and staging. Exp Ther Med 2015; 9: 905-8.

How to cite this article: Sak K. A hypothetical approach on gender differences in cancer diagnosis. J Transl Int Med 2019; 7: 90-2. 